\title{
ON ADDITIVE PREMIUM CALCULATION PRINCIPLES
}

\author{
Hans U. Gerber
}

\section{INTRODUCTION}

A premium calculation principle is a general rule that assigns a premium $P$ to any given risk $S$. Intuitively, $P$ is what the insurance carrier charges (apart from an expense allowance) for taking over the risk $S$ (see [3], p. 85-87). Mathematically, $S$ is a random variable, and $P$ depends on $S$ through its distribution function. The value of $P$ may be finite or infinite; in the latter case we speak of an uninsurable risk.

A premium calculation principle is called additive, if the premium assigned to the sum of two independent risks is the sum of the premiums that are assigned to the two risks individually. For example, the variance principle, $P=E[S]+\beta \operatorname{Var}[S](\beta>0)$, is additive, because the variance of the sum of independent random variables equals the sum of the variances. Additivity is a very desirable property, from a theoretical as well as from a practical point of view (as pointed out by Borch [2], p. 429).

The variance principle is not entirely satisfactory for various reasons. For one thing it does not take account of the skewness of $S$ (a risk whose distribution is skewed to the right seems to be more dangerous than one with a symmetrical distribution). Furthermore, it produces in some cases a premium $P$ that exceeds $S$ with probability one (example: $\beta=.3, S=0$ or Io, each with probability $\mathrm{I} / 2$ ).

In the sequel we shall focus our attention on the principle of zero utility (see [3], p. 86). Thus we assume that the insurance carrier bases his decisions on a utility function, i.e. a real valued function $u(x),-\infty<x<+\infty$, having the following properties:

(i) $u(x)$ is continuous and non-decreasing in $x$

(ii) the right side derivative $u^{\prime}(x)$ is non-increasing in $x$

(iii) $u(0)=0$

(iv) $u^{\prime}(0)=\mathbf{I}$. 
Any such utility function generates a premium calculation principle: $P$ is defined as the solution of the equation.

$$
E[u(P-S)]=0 .
$$

Thus $P$ is chosen such that the expected utility of the income $P-S$ is zero. Properties (i) and (ii) state that the insurance carrier is a risk averter, while (iii) and (iv) have the character of normalizing conditions. Because of (i) and (iv) the solution of (I) is unique. If (I) does not have a solution, $S$ is uninsurable.

\section{An Important Example: The Family of Exponential Utility Functions.}

In this section we consider utility functions of the form

$$
u(x)=(\mathrm{I} / a)\left(\mathrm{I}-e^{-a x}\right), \quad a \geq 0
$$

with the understanding that $u(x)=x$ for $a=0$. In this case we can solve (I) explicitly and find

$$
P=(\mathrm{I} / a) \ln E\left[e^{a S}\right]=(\mathrm{I} / a) \ln m(a)
$$

for $a>0$ and of course $P=E[S]$ for $a=0$, where $m($.$) is the$ momentgenerating function of $S$. Thus the premium is readily found whenever one knows the momentgenerating function of $S$. For example, in the case where $S$ has a normal distribution, $1 n$ $m(a)=a \mu+\left(a^{2} / 2\right) \sigma^{2}$, and (3) reduces to the variance principle with $\beta=(a / 2)$.

The parameter $a$ is a measure of the insurance carrier's risk aversion. The larger $a$, the larger is the premium for a given risk. This follows from the lemma below, in which $P(a)$ denotes the premium, as a function of $a$, for a fixed $S$.

Lemma: If $S$ is not a constant, and if $P(a)$ is finite for $0 \leq a<A$, then $P^{\prime}(a)>0$ for $0 \leq a<A$.

Proof: For $0<a<A$ we have

and

$$
P^{\prime}(a)=-(\mathrm{I} / a) P(a)+(\mathrm{I} / a) \frac{E\left[S e^{a S}\right]}{E\left[e^{a S}\right]}
$$

$$
(\mathrm{I} / a)\left(a^{2} P^{\prime}(a)\right)^{\prime}=\frac{E\left[S^{2} e^{a S}\right]}{E\left[e^{a} \bar{S}\right]}-\left(\frac{E\left[S e^{a S}\right]}{E\left[e^{a S}\right]}\right)^{2} .
$$


The last expression is positive (it is the variance of the Esscher transform of $S$ ). Therefore, $a^{2} P^{\prime}(a)$ is an increasing function in $a$, vanishing at $a=0$. Hence $a^{2} P^{\prime}(a)$ and $P^{\prime}(a)$ are positive for $o<a<A$. For $a=0$ one finds from (5) that $\left.P^{\prime}(0)=\frac{1}{2} \operatorname{Var}[S] .{ }^{*}\right)$

\section{Characterization of Additive Premium Calculation Principles}

Theorem: A utility function satisfying (i)-(iv) generates an additive premium calculation principle, if and only if it is of the form (2), i.e. if it is linear or exponential.

Proof: I) If $u(x)$ is of the form (2), then additivity holds

Let $a>0$ and suppose that $S_{1}, S_{2}$ are two independent, insurable risks with premiums $P_{1}, P_{2}$ (all other cases are trivial). Then the premium $P$ of $S_{1}+S_{2}$ is

$$
P=(\mathrm{I} / a) \ln E\left[e^{a\left(S_{1}+S_{2}\right)}\right]=(\mathrm{I} / a) \ln E\left[e^{a S_{1}}\right]+(\mathrm{I} / a) \ln E\left[a^{a S_{2}}\right]
$$

$$
=P_{1}+P_{2}
$$

2) If additivity holds, then $u(x)$ is of the form (2)

We prove this in two steps. First, we show that every Poisson distributed risk $S$ is insurable. Second, we derive a functional equation whose solutions are necessarily of the form (2).

Step a) Let $S_{q}$ be the Bernoulli risk with parameter $q$ : Prob $\left[S_{q}=\mathrm{o}\right]=\mathrm{I}-q, P\left[S_{q}=\mathrm{I}\right]=q$. Let $P_{q}$ denote the corresponding premium. By linearizing $u(x)$ to the right of $x=0$ and $x=-\mathrm{I}$, one recognizes that

$$
\lim _{q \rightarrow 0} \frac{P_{q}}{q}=-u(-\mathrm{I})
$$

Now let $S_{n q}$ denote the Binomial risk with parameters $n, q$ and let $P_{n q}$ be the corresponding premium. Because additivity holds, we have $P_{n q}=n P_{q}$. Thus (I) reads

$$
\sum_{k=0}^{n}\left(\begin{array}{l}
n \\
k
\end{array}\right) q^{k}(\mathrm{I}-q)^{n-k} u\left(n P_{q}-k\right)=0 .
$$

*) For a more extensive discussion of the exponential utility functions the reader is referred to [6]. Also, there he will find an alternative characterization of this family. 
Let $\lambda>0$ and let $n \rightarrow \infty, q \rightarrow 0$ with $n q=\lambda=$ constant. Taking this limit in (8), using (7), and dividing by $e^{-\lambda}$ we obtain

$$
\sum_{k=0}^{\infty} \frac{\lambda^{k}}{k !} u(-\lambda u(-\mathrm{I})-k)=0 .
$$

This shows that a Poisson distributed risk $S_{\lambda}$ (with parameter $\lambda$ ) is insurable and has premium $P_{\lambda}=-\lambda u(-\mathrm{x})$. From (9) we see also that the values of the function $u(x)$ for positive arguments are completely determined by the values for negative arguments.

Step b) For $z>0$ let $S_{z \lambda}=z S_{\lambda}$. By arguing as in a) one sees that $S_{z \lambda}$ is insurable and has premium $P_{z \lambda}=-\lambda u(-z)$. Consequently,

$$
\sum_{k=0}^{\infty} \frac{\lambda^{k}}{k !} u(-\lambda u(-z)-z k)=0
$$

for all $\lambda>0, z>0$. If we differentiate (Io) twice with respect to $\lambda$ from the right and set $\lambda=0$, we obtain the functional equation

$$
u(-2 z)-2 u(-z) u^{\prime}(-z)+u^{\prime \prime}(0) u(-z)^{2}=0
$$

for $z \geq 0$. Such an equation has a unique continuous solution (for $z>0$ ) satisfying the boundary condition $u(0)=0$. Hence $u(x)$ is necessarily of the form (2) with $a=-u^{\prime \prime}(0)$.

\section{Connection with the Collective Theory of Risk}

Encouraged by the result of the preceding section we shall from now on assume that an insurance carrier determines premiums on the basis of an exponential utility function. Thus suppose that a company consistently applies formula (3) for a certain constant value $a>0$. What is the resulting probability of ruin? Of course, the answer to this question depends on various factors, such as the initial surplus, the definition of ruin, and the distribution of the risks included in the portfolio. However, if we settle for the knowledge of the adjustment coefficient $R$ (in [I] it is called "insolvency constant'), and the resulting inequality for the probability of ruin, the answer is surprisingly simple: A comparison of (3) with formula (I2.I5), p. I44, in [I], reveals immediately that $R=a$. Thus if a company uses (3) consistently, it simply means that it choses the 
premiums in order to maintain a constant adjustment coefficient $R=$ a for the portfolio, which guarantees a probability of rwin less than $e^{-R x}(x=$ initial surplus).

\section{Credibility Theory}

In this section we assume that a risk is an element of a heterogeneous collective (see [3], chapter 4), each risk having an unknown label $\theta$. Let $S^{(\theta)}$ denote the risk for known value of $\theta$, and let $d W(\theta)$ be the distribution of $\theta$ in the collective. Applying the principle of zero utility with exponential utility function we obtain

$$
\begin{array}{rlrl}
P(\theta) & =(\mathbf{I} / a) \ln E\left[e^{a S^{(\theta)}}\right] & & \text { risk premium } \\
P & =(\mathrm{I} / a) \ln \left\{\int E\left[e^{a S^{(\theta)}}\right] d W(\theta)\right\} & \text { collective premium. }
\end{array}
$$

Notice that $P-\int P(\theta) d W(\theta)$ is positive (Jensen's inequality). This difference might be interpreted as the "fluctuation part" of the loading contained in $P$ (the part that is due to the randomness of $\theta$ ).

Credibility premiums are obtained by replacing $d W(\theta)$ in (I3) by a conditional distribution, determined by whatever information is available about a given risk. To illustrate this, let us discuss the special case "credibility for frequency".

Suppose that the distribution of $S$ is obtained by weighting compound Poisson distributions, where the weighting takes place with respect to the Poisson parameter $\theta \geq 0$ alone:

$$
\operatorname{Prob}[S \leq x]=\int_{0}^{\infty} \sum_{k=0}^{\infty} e^{-\theta} \frac{\theta^{k}}{k !} F^{* k}(x) d W(\theta) .
$$

Thus we assume that the underlying collective is homogeneous with respect to the claim amounts (their distribution $F(x)$ being independent of $\theta$ and of time), but inhomogeneous with respect to the claim numbers. The structure function $W(\theta)$ plays the role of a prior distribution on $\theta$. Furthermore suppose that $\phi=\int e^{a x} d F(x)$ is finite. From ( $\left.\mathrm{I}_{3}\right)$ and (I4) we find that the collective premium is

$$
P=(\mathrm{r} / a) \ln \left\{\int e^{\theta[\phi-1]} d W(\theta)\right\} .
$$

Now suppose that we are faced with a risk of which we know the total number of claims, say $N$, that occurred in the last $t$ periods 
(all other information is irrelevant under our assumptions). For the posteriori distribution $W(\theta ; t, N)$ we find that

$$
d W(\theta ; t, N) \sim e^{-\theta t} \theta^{N} d W(\theta) .
$$

By substituting this in ( 15 ) we obtain the credibility premium $P(t, N)$

$$
P(t, N)=(\mathrm{I} / a) \ln \frac{\int_{0}^{\infty} e^{\theta[\phi-1-t]} \theta^{N} d W(\theta) .}{\int_{0}^{\infty} e^{-\theta t} \theta^{N} d W(\theta)} .
$$

Let us now examine the question of insurability, i.e. the question whether the expressions in (I5) and (I7) are finite. Two cases have to be distinguished. Let

$$
w(t)=\int e^{\theta[\phi-1-t]} d W(\theta) .
$$

a) $w(t)<\infty$ for all $t \geq 0$.

In that case ( $\left.\mathrm{I}_{5}\right)$ and $\left(\mathrm{I}_{7}\right)$ are finite and the risk is insurable at any time.

b) $w(t)<\infty$ for $t>t_{0}>0, w(t)=\infty$ for $t<t_{0}$.

In this case the risk is not insurable before time $t_{0}$, and it is insurable any time after $t_{0}$ (no matter what the claim experience is). The question of insurability at time $t_{0}$ cannot be answered without making any further assumptions about $W(\theta)$. (Observe that the finiteness of $\phi$ implies that $w(t)$ is finite for large enough values of $t$ ).

Thus our results can be summarized as follows:

A risk may or may not be insurable at the beginning. But if it is insurable at one point in time, it will stay insurable forever.

In the special case where $W(\theta)$ is the Gamma-distribution with parameters $c$ and $\gamma$,

$$
d W(\theta) \sim e^{-c \theta} \theta^{\gamma-1} d \theta
$$

we obtain very neat formulas. A glance at (I6) shows that $W(\theta$; $t, N)$ is also a Gamma-distribution, with parameters $c_{t}=c+t$ and 
$\gamma_{N}=\gamma+N$. Using the momentgenerating function of the Gammadistribution, we obtain from (I5)

$$
P(t, N)=\frac{\gamma+N}{a}\left|\ln \left(\mathrm{I}-\frac{\phi-\mathrm{I}}{c+t}\right)\right| .
$$

So if $c>\phi-I$ the risk is insurable at any time. However, if $c<\phi-\mathrm{I}$, we find ourselves in case $b$ ), and the risk is insurable only after time $t_{0}=\phi-\mathrm{I}-c$.

\section{Cooperation of $n$ Insurance Companies}

In this section we utilize an idea of Buhlmann which he developed in connection with quadratic utility functions (see p. 197-200 of [3] or [4]). Here we assume that each of $n$ companies works with an exponential utility function, say with parameter $a_{i}=\left(\mathrm{I} / \alpha_{i}\right)$. Thus

$$
u_{i}(x)=\alpha_{i}-\alpha_{i} e^{x / \alpha_{i}} \quad(i=\mathrm{I}, 2, \ldots, n)
$$

Let $S$ be an arbitrary risk. How should the $n$ companies split up the risk among themselves in order to be as competitive as possible, i.e. in order to minimize the total premium?

Let $f_{i}(S)$ be the share that is taken over by company no. $i$. Obviously we must have $\sum_{i=1}^{n} f_{i}(S)=S$ for all outcomes of $S$. The premium $P_{i}$ of company $i$ is the solution of $E\left[u_{i}\left(P_{i}-f_{i}\right)\right]=0$, and we want to minimize $P=P_{1}+P_{2}+\ldots+P_{n}$. The arrangement $\left\{P_{1}-f_{1}(S), P_{2}-f_{2}(S), \ldots, P_{n}-f_{n}(S)\right\}$ must be a Pareto optimal partition of $P-S$ (if it was not Pareto optimal we could replace it by a partition.

$\left\{\tilde{P}_{1}-\tilde{f}_{1}, \tilde{P}_{2}-\tilde{f}_{2}, \ldots, \tilde{P}_{n}-\tilde{f}_{n}\right\}$ such that $E\left[u_{1}\left(\tilde{P}_{1}-\tilde{f}_{1}\right)\right]>0$ and $E\left[u_{i}\left(\tilde{P}_{i}-\tilde{f}_{i}\right)\right] \geq 0$ for $i=2, \ldots, n$. Thus $\tilde{P_{1}}$, and hereby $P$, could be reduced). In the case of exponential utility functions, all the Pareto optimal partitions of $P-S$ are of the form $\left\{k_{1}-\zeta_{1} S, k_{2}-\zeta_{2} S, \ldots, k_{n}-\zeta_{n} S\right\}$ where $k_{1}, k_{2}, \ldots, k_{n}$ are arbitrary constants with $\sum_{i=1}^{n} k_{i}=P$ and

$$
\zeta_{i}=\left(\alpha_{i} / \alpha\right) \text { with } \alpha=\alpha_{1}+\alpha_{2}+\ldots+\alpha_{n}
$$


as shown on p. $202[7]$ (it might also be derived as a consequence of Borch's theorem, see [2], [3], or [5]). In our context, the $k_{i}$ 's are of course not arbitrary, but $k_{i}=P_{i}$ and

$$
P_{i}=\alpha_{i} \ln E\left[e^{(1 / \alpha) S}\right]
$$

Thus the minimal total premium of $S$ becomes

$$
P=\alpha \ln E\left[e^{(1 / \alpha) S}\right]
$$

So we find: If the $n$ companies cooperate in order to minimize the total premium, company no. $i$ will take over $\left(\alpha_{i} / \alpha\right) S$, and the resulting total premium can be interpreted as the premium of one single company working with an exponential utility function with parameter $\propto$. This result corresponds to Buhlmann's result concerning additivity of levels of saturation (see [3], p. I97-200) in the case of quadratic utility functions.

\section{REFERENCES}

[i] R. Beard, T. Pentikatnen, E. Pesonen, Risk Theory, Methuen, London, 1969.

[2] K. Borch, "Equilibrium in a reinsurance market", Econometrica 30 (I962), 424-444.

[3] H. Buhlmann, Mathematical Methods in Risk Theory, Springer, New York, 1970.

[4] H. Buhlmann, "Individual, cooperative and competitive pricing of risks" Risk and uncertainty (Conference IEA, Smolenice) ed. by K. Borch and J. Mossin. Macmillan, London, r968, p. 265-282.

[5] W. Dumouchel, "The Pareto-optimality of a n-company reinsurance treaty" Skandinavisk Aktuarietidskrift 5I (I968), r65-I 7o.

[6] C. DE FERRA, "Considerazioni sulle funzioni di utilità in connessione con la teoria del rischio". Giornale dell' Istituto Italiano degli Attuari 27 (I964), 5I-7o.

[7] H. Raiffa, Decision Analysis, Addison-Wesley, Reading, 1968. 